\title{
Conditional Diagnosability of the Locally Twisted Cubes under the PMC Model*
}

\author{
Ruitao Feng ${ }^{1}$, Genqing Bian ${ }^{1}$, Xinke Wang ${ }^{2}$ \\ ${ }^{1}$ School of Information and Control Engineering, Xi' an University of Architecture \\ and Technology, Xi'an, China \\ ${ }^{2}$ Department of Mathematics, Xidian University, Xi'an, China \\ E-mail: \{qinshiyue2003,bgq_00\}@163.com,wxk1383@126.com \\ Received August 11, 2011; revised September 20, 2011; accepted September 30, 2011
}

\begin{abstract}
In a multiprocessor systems, it is important to local and to replace the faulty processors to maintain systempsilas high reliability. The fault diagnosis, which is the process of identifying fault processors in a multiprocessor system through testing. The conditional diagnosis requires that for each processor $\mathrm{u}$ in a system, all the processors that are directly connected to u do not fail at the same time. In this paper, we study the conditional diagnosability of the n-dimensional locally twisted cubes. After showing some properties of the locally twisted cubes, we prove that it under the PMC model is $4 n-7$ for $n \geq 5$.
\end{abstract}

Keywords: Locally Twisted Cubes, Diagnosability, Conditional Diagnosability, PMC Mode

\section{Introduction}

In recent years, the number of the processors in a multiprocessor system increases as fast as the technology development. Thus some processors may fail in such a multiprocessor operating system. So locating the faulty processors is important for system maintenance and dependable computing. System level diagnosis is an important approach for fault diagnosis in a multiprocessor system. Many different models for system level diagnosis in multiprocessor systems have been proposed, e.g., the PMC (the Perfect Minicomputer Corporation) model [1], the comparison model [2] and the BGM model [3]. So far, the well-studied mode is the PMC model introduced by Preparata, Metze, and Chien [1].

A multiprocessor system is an interconnected collection of processors and can be represented by an undirected graph $G=(V, E)$, where each vertex of the vertex set $\mathrm{V}$ represents a processor and each edge of the edge set $\mathrm{E}$ represents a communication link between a pair of processors. Two processors interact with each other by sending messages over the communication link. Under the PMC model, two processors can test each other if and only if there is a link between them. The processor which

\footnotetext{
* The paper supported by the National Natural Science Foundation of China (No.61073196) and Natural Science Research Foundation of Shanxi Province, China (No. 2011JM8026).
}

tests the status of the other is called a tester. It is assumed that the test result is reliable if and only if the tester is fault free; otherwise, the test result is unreliable. The collection of all test results is called a syndrome $\sigma \cdot r(u, v)$ denotes the test result of processor u testing processor v. If $\mathrm{v}$ pass the test executed by $\mathrm{u}, r(u, v)=0$; otherwise, $r(u$, $v)=1$. Table 1 shows all possible test results of the test $r(u, v)$.

For a given syndrome $\sigma$, a subset of vertices $F \subseteq V$ is said to be consistent with $\sigma$ if $\sigma$ can arise from the circumstance that all nodes in $\mathrm{F}$ are faulty and all nodes in $V-F$ are fault free. It is worth pointing out that a given set $F$ of faulty vertices may be consistent with different syndromes. Let $\sigma(F)$ be the set containing all syndromes which can be produced by $F$. Two distinct sets $F_{1}, F_{2} \subseteq V$ are said to be distinguishable if $\sigma\left(F_{1}\right) \cap \sigma\left(F_{2}\right)=\varnothing$ otherwise, $F_{1}, F_{2}$ are said to be indistinguishable.

Table 1. Test results.

\begin{tabular}{ccc}
\hline$u$ & $v$ & $r(u, v)$ \\
\hline Fault-free & Fault-free & 0 \\
Fault-free & Fault & 1 \\
Fault & Fault-free & 0 or 1 \\
Fault & Fault & 0 or 1 \\
\hline
\end{tabular}


A system is said to be $t$-diagnosable if given a syndrome $\sigma$, all processors can correctly be identified faulty or faulty free, provided that the number of faulty processors present in the system does not exceed $t$. The diagnosability of a system is the maximal number of faulty processors that the system can guarantee to diagnose. The diagnosability of some interconnection networks have been discussed under the PMC model, see [4-6].

Lai et al. in [7] introduced conditional diagnosability by restricting that for each processor $u$ in a system, all processors adjacent to $u$ are not faulty at the same time, and showed that conditional diagnosability of the n-dimensional hypercube $\left(Q_{n}\right)$ is $4 n-7$ for $n \geq 5$, which is about four times as large as its classical diagnosability [8]. Zhu et al. in [9] presented that under PMC-model the conditional diagnosability of FQn $\left(t_{c}\left(F Q_{n}\right)\right)$ was $4 \mathrm{n}-3$ when $\mathrm{n} \geq 5$ or $\mathrm{n}>8 ; t_{c}\left(F Q_{3}\right)=3, t_{c}\left(F Q_{4}\right)=7$.

In recent years, conditional diagnosability of several interconnection networks has also been explored under the PMC model [7,9-12].

In the paper, we prove that conditional diagnosability of locally twisted cubes under the $P M C$ model is $t_{c}\left(L T Q_{n}\right)$ $=4 n-7$ for $n \geq 5$.

The rest of paper is organized as follows: Preliminary knowledge is provided in Section 2; The main results of this paper are presented and proven in Section 3; The conclusions are made in Section 4.

\section{Preliminaries}

For all the terminologies and notations not defined here, we follow [13]. For a graph $G=(V, E)$ and $S \subset V(G)$ or $S \subset G$, we use $N_{G}(S)$ to denote the set of neighboring vertices of $S$ in $G-S$, when it is easy to know from the context what $G$ denotes, it is usually simplified with $N(S)$. We use $A_{G}(S)$ to denote the union of $S$ and $N_{G}(S)$. And similarly $A_{G}(S)$ can be simplified with $A(S)$.

That is, $N_{G}(S)=\{v \in V(G)-S \mid \exists u \in \mathrm{S}$ such that $(u, v) \in$ $E(G)\}, A_{G}(S)=N_{G}(S) \cup S$.

We use $d_{G}(u)$ to denote the degree of $\mathrm{u}$ in $\mathrm{G}$ and $d_{G}(u)$ can be simplified with $d(u)$.

Definition 1. [14] For $n \geq 2$, an $n$-dimensional locally twisted cube, denoted by $L T Q_{n}$, is defined recursively as follows:

1) $\mathrm{LTQ}_{2}$ is a graph consisting of four nodes labeled with $00,01,10$ and 11 , respectively, connected by four edges $\{00,01\},\{01,11\},\{11,10\}$ and $\{10,00\}$.

2) For $n \geq 3, L T Q_{n}$ is built from two disjoint copies of $L T Q_{n-1}$ according to the following steps: Let $0 L T Q_{n-1}$ denote the graph obtained from one copy of $L T Q_{n-1}$ by prefixing the label of each node with 0 . Let $1 L T Q_{n-1}$ denote the graph obtained from the other copy of $L T Q_{n-1}$ by prefixing the label of each node with 1 . Connect each node $0 x_{2} x_{3} \cdots x_{n}$ of 0LTQn 1 to the node $1\left(x_{2}+x_{n}\right) x_{3} \cdots x_{n}$ of $1 L T Q_{n} 1$ with an edge, where “+” represents the modulo 2 addition.

Figure 1 shows two examples of locally twisted cubes. The locally twisted cubes can also be equivalently defined in the following non-recursive fashion.

Definition 2. [14] For $n \geq 2$, the $n$-dimensional locally twisted cube, $L T Q_{n}$, is a graph with $\{0,1\}^{n}$ as the node set. Two nodes $x=x_{1} x_{2} \cdots x_{n}$ and $y=y_{1} y_{2} \cdots y_{n}$ of $L T Q_{n}$ are adjacent if and only if either one of the following conditions are satisfied.

1) $x_{i}=\overline{y_{i}}$ and $x_{i+1}=y_{i+1}+y_{n}$ for some $1 \leq \mathrm{i} \leq \mathrm{n}-2$, and $x_{j}=y_{j}$ for all the remaining bits;

2) $x_{i}=\bar{y}_{i}$ for $i \in\{n-1, n\}$, and $x_{j}=y_{j}$ for all the remaining bits.

The definition of the conditional diagnosability is as follows.

Definition 3. [7] A faulty set $F \subseteq V$ is called a conditional faulty set if $N(v) \not \subset F$ for any vertex $v \in V$. A system $G(V, E)$ is conditionally $t$-diagnosable if $\mathrm{F}_{1}$ and $\mathrm{F}_{2}$ are distinguishable, for each pair of conditional faulty sets $F_{1}, F_{2} \subseteq V$, and $F_{1} \neq F_{2}$ with $\left|F_{1}\right| ;\left|F_{2}\right| \leq \mathrm{t}$. Conditional diagnosability of a system $G$, written as $t_{c}(G)$ is defined to be the maximum value of $t$ such that $G$ is conditionally $t$-diagnosable.

Let $F_{1}, F_{2}$ be two distinct sets, the symmetric difference of $F_{1}$ and $F_{2}$ is denoted by $F_{1} \Delta F_{2}$, that is, $F_{1} \Delta F_{2}=$ $\left(F_{1}-F_{2}\right) \cup\left(F_{2}-F_{1}\right)$. The following lemma proposed by Dahbura and Masson [15] gives a necessary and sufficient condition for a system to be $t$-diagnosable.

Lemma 1. [16] A system $G(V, E)$ is $t$-diagnosable if and only if, for each pair $F, F_{2} \subset V$ with $\left|F_{1}\right|,\left|F_{2}\right| \leq t$ and $F_{1} \neq F_{2}$, there is at least one test from $V-F_{1} \cup F_{2}$ to $F_{1} \Delta F_{2}$.

Lemma 2. [14] $k\left(L T Q_{n}\right)=n$ for $n \geq 2$.

Lemma 3. [17] $k\left(L T Q_{n}\right)=2 n-2$ for $n \geq 3$.

Lemma 4. [17] Let $S$ be a set of vertices $S \subset V\left(L T Q_{n}\right)$ with $|S|=n$, if $L T Q_{n}-S$ is disconnected, there exists $a$ vertex $u \in V\left(V\left(L T Q_{n}\right)\right)$ such that $N(u)=S$ for $n \geq 2$.

The following lemma is derived based on $[18,19]$.
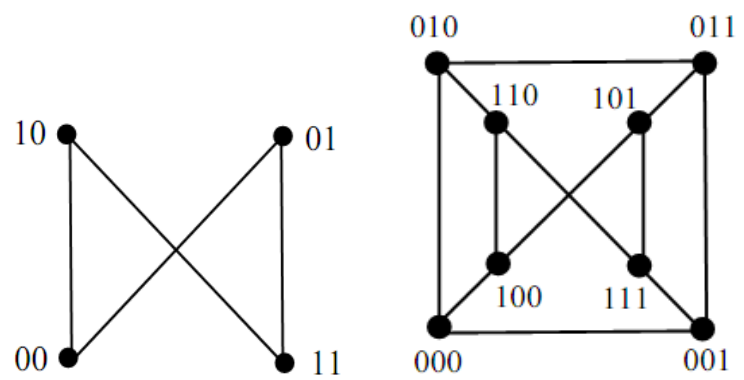

Figure 1. Example of $L T Q_{n}: L T Q_{2}$ and $L T Q_{3}$. 
Lemma 5. Let $F$ be a subgraph of $L T Q_{n}$ with $4 \leq|V(F)| \leq 3 n-5$, we have $\left|N_{L T Q_{n}}(F)\right| \geq 4 n-8$.

\section{Conditionally Diagnosability}

Lemma 6. Let $S$ be a set of vertices $S \subset V\left(L T Q_{n}\right)$ and $n \geq$ 3. Suppose that $L T Q_{n}-S$ is disconnected. The following two conditions hold:

(1) $|S| \geq n$;

(2) If $n \leq|S| \leq 2 n-3$, then $L T Q_{n}-S$ has exactly two components, one is trivial and the other is nontrivial. The nontrivial component of $L T Q_{n}-S$ contains $2^{n}-|S|-1$ vertices.

Proof: By lemma $2 k\left(L T Q_{n}\right)=n$, so condition (1) holds. We need to prove that condition (2) is true. Since $L T Q_{n}-S$ is disconnected, there are at least two components in $L T Q_{n}-S$. We will prove that |S्f $2 n-2$ when $L T Q_{n}-S$ contains at least two trivial components or two nontrivial components. It implies that $n \leq|S|$ $\leq 2 n-3$ when $L T Q_{n}-S$ contains a trivial components and nontrivial components.

Case 1. $L T Q_{n}-S$ contains at least two trivial components. Let $u_{1}, u_{2} \in V\left(L T Q_{n}\right)$ and $\left\{u_{1}\right\},\left\{u_{2}\right\}$ be two trivial components. Then $N\left(u_{1}\right) \subset \mathrm{S}$ and $N\left(u_{2}\right) \subset \mathrm{S}$. Since any two distinct vertices of a $L T Q_{n}$ have at most two common neighbors, we have $\left|N\left(V_{1}\right) \cap\right| N\left(V_{2}\right) \mid \leq 2$.

Hence, $|S| \geq\left|N\left(V_{1}\right)\right|+\left|N\left(V_{2}\right)\right|-\left|N\left(V_{1}\right) \bigcap N\left(V_{2}\right)\right| \geq 2 n$ $+2 n-2=2(2 n-1)$.

Case 2. $L T Q_{n}-S$ contains at least two nontrivial components. We prove condition (2) by induction on $\mathrm{n}$. Suppose $n \leq|S| \leq 2 n-3$, it is easy to see that $|S|=3$ for $n$ $=3$. The connectivity of $L T Q_{3}$ is 3 . By Lemma 4 , there exist a vertex $u \in V\left(L T Q_{3}\right)$ such that $S=N(u)$ Thus $L T Q_{3}-S$ has exactly two components: one is trivial and the other is nontrivial. Therefore, if $L T Q_{3}-S$ has at least two nontrivial components, $|S| \geq 2 n-2$, where $n=3$. Assume that the result holds for all $n-1, n-1 \geq 3$. In the following we show that it holds for $n$.

Let $S_{0}=S \cap \mathrm{V}\left(0 L T Q_{n-1}\right)$ and $S_{1}=S \cap V\left(1 L T Q_{n-1}\right)$, $F$ and $F^{\prime}$ be two nontrivial component of $L T Q_{n}-S$. So $|V(F)| \geq 2$ and $\left|\mathrm{V}\left(\mathrm{F}^{\prime}\right)\right| \geq 2$.

We consider the following three cases:

Case 2.1. $F, \mathrm{~F}^{\prime} \subseteq 0 L T Q_{n-1}$ or $F, F^{\prime} \subseteq 1 L T Q_{n-1}$. Without loss of generality, let $\mathrm{F}, \mathrm{F} \subseteq O L T Q_{n-1}$, then $0 L T Q_{n-1}-S_{0}$ is disconnected and $\left|S_{1}\right| \geq|F|+\left|F^{\prime}\right| \geq 4$. So $\left|\mathrm{S}_{0}\right| \geq k_{2}=2 n-$ 4 by lemma 3. Thus $|S|=\left|S_{0}\right|+\left|S_{1}\right| \geq 2 n-2$.

Case 2.2. $F \subseteq 0 L T Q_{n-1}$ and $F^{\prime} \subseteq 1 L T Q_{n-1}$, or $F^{\prime} \subseteq$ $1 L T Q_{n-1}$ and $\mathrm{F} \subseteq 1 L T Q_{n-1}$. Without loss of generality, let $F \subseteq 0 L T Q_{n-1}$ and $F^{\prime} \subseteq 1 L T Q_{n-1}$. If both $0 L T Q_{n-1}-S_{0}$ and $1 L T Q_{n-1}-S_{1}$ are connected, then $\left|S_{0}\right| \geq 2 n-4$ and $S_{1} \mid \geq$ $2 n-4$. So $|S|=\left|S_{0}\right|+\left|S_{1}\right| \geq 2 n-4+2 n-4 \geq 2 n-2$ for $\mathrm{n}$ $\geq 3$. If exactly one of $0 L T Q_{n-1}-S_{0}$ and $1 L T Q_{n-1}-S_{1}$ is disconnected, let $0 L T Q_{n-1}-S_{0}$ be disconnected, then $N_{0 L T Q_{n}}(F) \subseteq S_{0}$. So

$$
|S| \geq \mathbb{Q}_{0 L T Q_{n}}(F 2)+2=\mathbb{Z}_{-}+=n-
$$

Case2.3. $0 L T Q_{n-1} \cap F \neq \varnothing$ and $1 L T Q_{n-1} \cap F \neq \varnothing$, or $0 L T Q_{n-1} \cap F^{\prime} \neq \varnothing$ and $1 L T Q_{n-1} \cap F^{\prime} \neq \varnothing$. Without loss of generality, let $0 L T Q_{n-1} \cap F \neq \varnothing$ and $1 L T Q_{n-1} \cap F \neq \varnothing$. Since there is another component $F^{\prime}$ of $L T Q_{n}-S$, at least one of the two graphs $0 L T Q_{n-1}-$ $S_{0}$ and $1 L T Q_{n-1}-S_{1}$ is disconnected. So we drive the result by consider two Subcase.

Case 2.3.1. Both $0 L T Q_{n-1}-S_{0}$ and $1 L T Q_{n-1}-S_{1}$ are disconnected. Since $k\left(L T Q_{n-1}\right)=n-1,\left|S_{0}\right| \geq n-1$ and

$\left|S_{1}\right| \geq n-1$. Then $|S|=\left|S_{0}\right|+\left|S_{1}\right| \geq 2 n-2$.

Case 2.3.2. Exactly one of the two subgraphs $0 L T Q_{n-1}$ $-S_{0}$ and $1 L T Q_{n-1}-S_{1}$ is disconnected. Without loss of generality, assume that $0 L T Q_{n-1}-S_{0}$ is connected and $1 L T Q_{n-1}-S_{1}$ is disconnected. Then $\left|S_{1}\right| \geq \mathrm{n}-1$ and $N_{\text {OLTQn }}(F) \subseteq S_{0}$. Hence, $\left|S_{0}\right| \geq\left|\mathrm{V}\left(F^{\prime}\right)\right| \geq 2$. If $\left|S_{1}\right| \geq 2 n-4$, then $|S|=\left|S_{0}\right|+\left|S_{1}\right| \geq 2+(2 n-4)=2 n-2$. Otherwise, $n$ $-2 \leq\left|S_{1}\right| \leq 2 n-5$. By induction hypothesis, $1 L T Q_{n-1}-S_{1}$ has exactly two components: one is trivial and the other is nontrivial. We know that $1 L T Q_{n-1} \cap F$ and $F^{\prime}$ are two components of $1 L T Q_{n-1}-S_{1}$, and $F^{\prime}$ is a nontrivial component. Thus $1 L T Q_{n-1} \cap F$ must be a trivial component of $11 L T Q_{n-1}-S_{1}$, and $\left|V\left(F^{\prime}\right)\right|=2^{n-1}-\left|S_{1}\right|-1$. Note that $N_{0 L T Q n}\left(F^{\prime}\right) \subseteq S_{0}$. Hence, $|\mathrm{S}|=\left|S_{0}\right|+\left|S_{1}\right| \geq\left|V\left(F^{\prime}\right)\right|+\left|S_{1}\right|$ $=2^{n-1}-\left|S_{1}\right|-1+\left|S_{1}\right| \geq 2 n-2$ for $n \geq 4$.

Consequently, condition (2) holds.

Lemma 7: Let $S$ be a set of vertices $S \subset V\left(L T Q_{n}\right)$ and $\mathrm{n} \geq 5$. Suppose that $L T Q_{n}-S$ is disconnected and every component of $L T Q_{n}-S$ is nontrivial, and there exists one component $F$ of $L T Q_{n}-S$ such that $d_{F}(v) \geq 2$ for any vertex $v \in F$. Then one of the following two conditions must hold:

(1) $|S| \geq 4 n-8$;

(2) $|V(F)| \geq 4 n-9$.

Proof: Let $F_{0}=0 L T Q_{n-1} \cap F, F_{1}=1 L T Q_{n-1} \cap F$, $S_{0} .=S \cap V\left(0 L T Q_{n-1}\right)$ and $S_{1}=S \cap V\left(1 L T Q_{n-1}\right)$. We consider two cases: (a) $F \subset 0 L T Q_{n-1}$ or $F \subset 1 L T Q_{n-1}$. (b) $0 L T Q_{n-1} \cap F \neq \varnothing$ and $1 L T Q_{n-1} \cap F \neq \varnothing$.

Case 1. $F \subset 0 L T Q_{n-1}$ or $F \subset 1 L T Q_{n-1}$. Without loss of generality, let $F \subset 0 L T Q_{n-1}$. Then $F \subset S_{1}$. In the following we consider two cases.

Case 1.1. $0 L T Q_{n-1}-F$ is connected. Then $|S|=\left|S_{0}\right|+\left|S_{1}\right|$ $\geq\left|S_{0}\right|+|V(F)|=2^{n-1} \geq 2 n-2$ for $n \geq 4$ and conditional (a) holds.

Case 1.2. $0 L T Q_{n-1}-F$ is disconnected. If $4 \leq|V(F)| \leq$ $3 n-5$, by Lemma 5, we have $\left|\mathrm{S}_{0}\right| \geq\left|N_{L T Q_{\eta}}(F)\right| \geq 4 n-$ 8.Therefore, $|S| \geq 4 n-8$ and conditional (a) holds. If $3 n$ $-4 \leq|V(F)| \leq 4 n-10$, then $\left|S_{0}\right| \geq n-1$ since $0 L T Q_{n-1}-F$ 
is disconnected and $\left|S_{1}\right| \geq|V(F)| \geq 3 n-4$. Thus $|S|=\left|S_{0}\right|+$ $\left|S_{1}\right| \geq n-1+3 n-4=4 n-5$ and conditional (a) holds. Otherwise, $|V(F)| \geq 4 n-9$ and conditional (b) holds.

Case 2. $0 L T Q_{n-1} \cap F \neq \varnothing$ and $1 L T Q_{n-1} \cap F \neq \varnothing$. Since every vertex $x$ in $F_{0}$ (resp. $y$ in $F_{1}$ ) has at most one neighbor in $F_{1}$ (resp. $F_{0}$ ), we have $d_{F_{0}}(x) \geq 1$ and $d_{F_{1}}(x)$. Since $L T Q_{n}-S$ is disconnected, there are at least two components in $L T Q_{n}-S$. At least one of the two graphs $0 L T Q_{n-1}-S_{0}$ and $1 L T Q_{n-1}-S_{1}$ is disconnected since both $L T Q_{n}$ and $L T Q_{n-1}$ contain some non-empty part of the component $F$.

In the following we drive the result by consider two cases.

Case 2.1. Exactly one of the two graphs $0 L T Q_{n-1}-S_{0}$ and $1 L T Q_{n-1}-S_{1}$ is disconnected. Without loss of generality, assume that $0 L T Q_{n-1}-S_{0}$ is connected and $1 L T Q_{n-1}-S_{1}$ is disconnected. Let $F^{\prime}$ be another non-trivial component of $L T Q_{n}-S$ other than $F$. Then $F^{\prime} \subset\left(1 L T Q_{n-1}-S_{1}\right)$ and $N_{0 L T Q_{n-1}}\left(F^{\prime}\right) \subset S_{0}$. Note that both $F^{\prime}$ and $F_{1}$ are nontrivial component. By Lemma 6, $\left|S_{1}\right| \geq 2 n-4$. If $j\left|S_{0}\right| \geq 2 n-4$, then $|S|=\left|S_{0}\right|+\left|S_{1}\right| \geq 4 n$ -8 and condition (1) holds. Otherwise, $\left|S_{0}\right| \leq 2 n-5$. Then $\left|V\left(F_{0}\right)\right|=2^{n-1}-\left|S_{0}\right|$ since $V\left(L T Q_{n-1}\right)=S_{0} \cup V\left(F_{0}\right)$.

Thereby, $|V(F)|=\left|V\left(F_{0}\right)\right|+\left|V\left(F_{1}\right)\right| \geq\left(2^{n-1}-(2 n-5)\right)+$ $2 \geq 4 n-9$ for $n \geq 4$ and condition (2) holds.

$0 L T Q_{n-1}-S_{0}$ and $1 L T Q_{n-1}-S_{1}$ are disconnected. We consider the following three subcases.

Case 2.1.1. $\left|S_{0}\right| \geq 2 n-4$ and $\left|S_{1}\right| \geq 2 n-4$.Clearly, $S \mid=$ $\left|S_{0}\right|+\left|S_{1}\right| \geq 8 n-8$. Therefore, condition (1) holds.

Case 2.2.2. Either $n-1 \leq\left|S_{0}\right| \leq 2 n-5,\left|S_{1}\right| \geq 2 n-4$ or $\left|S_{0}\right| \geq 2 n-4, n-1 \leq\left|S_{1}\right| \leq 2 n-5$. Without loss of generality, assume that $S_{0}|\geq 2 n-4, n-1 \leq| S_{1} \mid \leq 2 n-5$. Then we have $\left|V\left(F_{1}\right)\right|=2^{n-1}-\left|S_{1}\right|-1$ by the lemma 6 . Since $d_{F_{0}}(u) \geq 1$ for any vertex $u \in V\left(F_{0}\right),\left|V\left(F_{0}\right)\right| \geq 2$. Thus, $|V(F)|=\left|V\left(F_{0}\right)\right|+\left|V\left(F_{1}\right)\right| \geq 2+\left(2^{n-1}-2 n-4\right) \geq$ $4 n-9$ for $n \geq 5$. Hence, condition (2) holds.

Case 2.2.3. $n-1 \leq\left|S_{0}\right| \leq 2 n-5$ and $n-1 \leq\left|S_{1}\right| \leq 2 n-$ 5. By the lemma 6, we have $\left|V\left(F_{0}\right)\right|=2^{n-1}-\left|S_{0}\right|-1$ and $\mid V$ $\left(F_{1}\right)\left|=2^{n-1}-\right| S_{1} \mid-1$. So $|V(F)|=\left|V\left(F_{0}\right)\right|+\left|V\left(F_{1}\right)\right|=2^{n}-$ $|S|-2$. If $|S| \geq 4 n-8$, then condition (1) holds. Otherwise, $|S| \leq 4 n-9$, then $|V(F)|=2^{n}-(4 n-9)-2 \geq 4 n-9$ for $n$ $\geq 4$. Hence, condition (2) holds.

Consequently, the lemma holds.

Theorem 1. Let $F_{1}, F_{2} \subset V\left(L T Q_{n}\right)$ be two indistinguishable conditional faulty sets, then either $\left|F_{1}\right| \geq 4 n-6$ or $\left|F_{2}\right| \geq 4 \mathrm{n}-6$ for $n \geq 5$.

Proof: Let $S=F_{1} \cap F_{2}$, according to $L T Q_{n}-S$ is connected or not, we consider the following two cases.

Case 1. $L T Q_{n}-\mathrm{S}$ is connected. We assert that $F_{0} \Delta F_{1}=$ $V\left(L T Q_{n}\right)-$ S. Otherwise, suppose $u \in$

$V\left(L T Q_{n}-S\right)-F_{1} \Delta F_{2}=V\left(L T Q_{n}\right)-F_{1} \cup F_{2}$. Then $u$ is connected to $F_{1} \Delta F_{2}$ since $L T Q_{n}-S$ is connected. That is, there is an edge between $F_{1} \Delta F_{2}$ and $V-F_{1} \cup F_{2}$. This is a contradiction to the fact $F_{1}$ and $F_{2}$ are an indistinguishable. Since $\left|F_{1}\right|+\left|F_{2}\right|=\left|F_{1}\right| \Delta\left|F_{2}\right|=\left|V\left(L T Q_{n}\right)\right|=2^{n} \geq$ $8 n-13$ for $n \geq 5$, either $\left|F_{1}\right| \geq 4 n-6$ or $\left|F_{2}\right| \geq 4 n-6$. Then the result follows.

Case 2. $L T Q_{n}-S$ is disconnected. Since $F_{1}$ and $F_{2}$ is indistinguishable, there is no edge between $F_{1} \Delta F_{2}$ and $V$ $\left(L T Q_{n}\right)-F_{1} \cup F_{2}$ by Lemma 1 .That is, for any vertex $u$ $\in F_{1} \Delta F_{2}, \quad N_{L T Q_{n}}(u) \subset F_{1} \cup F_{2}$. Since both $F_{1}$ and $F_{2}$ are conditional faulty set, $N_{L T Q_{n}}(u) \not \subset F_{1}$

and $N_{L T Q_{n}}(u) \not \subset F_{2}$. So $\left|N_{L T Q_{n}}(u) \cap\left(F_{2}-F_{1}\right)\right|$ and $\left|N_{L T Q_{n}}(u) \cap\left(F_{2}-F_{1}\right)\right| \geq 1$.

Thus for any vertex $u \in F_{1} \Delta F_{2},\left|N_{F_{1} \Delta F_{2}}(u)\right| \geq 2$. So $L T Q_{n}-S$ has a component $P$ with $V(P) \subset F_{1} \Delta F_{2}$ such that $d_{P}(u) \geq 2$ for any vertex $\mathrm{u} \in V(P)$. By Lemma 7 , we have $|S| \geq 4 n-8$ or $|V(P)| \geq 4 n-9$ for $n \geq 5$. So we consider the following two subcases.

Case 2.1. $|S| \geq 4 n-8$. Let $C$ be a cycle in $P$. Since $d_{P}(u) \geq 2$ for each vertex $u \in V(F)$, and $V\left(L T Q_{n}\right) \geq 4$, the cycle $C$ of length is not less than 4 . Because $\mathrm{V}(\mathrm{C}) \subset$ $\mathrm{V}(\mathrm{P}) \subset F_{1} \Delta F_{2}$, either $\left|F_{1}-F_{2}\right| \geq 2$ or $\left|F_{2}-F_{1}\right| \geq 2$. Thereby, either $\left|F_{1}\right|=|S|+\left|F_{1}-F_{2}\right| \geq 4 n-6$ or $\left|F_{2}\right|=|S|+\mid F_{1}$ $-F_{2} \mid \geq 4 n-6$.

Case 2.2. $|V(P)| \geq 4 n-9$. Since $|V(P)| \geq 4 n-9$ and

$\mathrm{V}(\mathrm{P}) \subset F_{1} \Delta F_{2}$, either $\left|F_{1}-F_{2}\right| \geq 2 n-4$ or $\left|F_{2}-F_{1}\right| \geq$ $2 n-4$. And since there is no isolated vertex in $L T Q_{n}$ (both $F_{1}$ and $F_{2}$ are conditional faulty set) and $L T Q_{n}-\mathrm{S}$ is disconnected, $\mid S \notin 2 n-2$ by lemma 3. Thereby, either $\left|F_{1}\right|=|\mathrm{S}|+\left|F_{1}-F_{2}\right| \geq 4 n-6$ or $\left|F_{2}\right|=|\mathrm{S}|+\left|F_{2}-F_{1}\right| \geq$ $4 n-6$.

Consequently, the theorem holds.

The theorem 1 shows that the conditional diagnosability of $L T Q_{n}$ is not less than $4 n-7$ for $n \geq 5$. In the following we will show that the conditional diagnosability of $L T Q_{n}$ is not more than $4 n-7$ for $n \geq 5$.

Theorem 2. $t_{c}\left(L T Q_{n}\right) \leq 4 n-7$ for $n \geq 3$.

Proof: (See Figure 2) Let $C=\left(u_{1}, u_{2}, u_{3}, u_{4}\right)$ be a cycle of length 4 in $L T Q_{n} . u_{1}, u_{2}, u_{3}, u_{4}$ are the four consecutively vertices in the cycle $C$. Let $F_{1}=N_{L T Q_{n}}(C) \cup$ $\left\{u_{1}, u_{2}\right\}$ and $F_{2}=N_{L T Q_{n}}(C) \cup\left\{u_{3}, u_{4}\right\}$. It is easy to verify that $F_{1}$ and $F_{2}$ are two indistinguishable conditional faulty

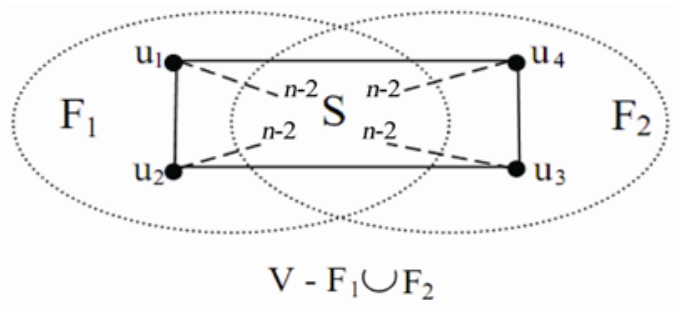

Figure 2. An illustration of the proof of Theorem 2. 
set. It is easy to see that there exists no triangle in $L T Q_{n}$ and any two distinct vertices in $L T Q_{n}$ have at most two common neighbors. Thus we have $\left|F_{1} \cap F_{2}\right|=N_{L T Q_{n}}(C)$ $=4 n-8$ and $\left|F_{1}-F_{2}\right|=\left|F_{2}-F_{1}\right|$. So $\left|F_{1}\right|=\left|F_{2}\right|=4 n-6$. Hence, $L T Q_{n}$ is not conditionally $(4 n-6)$ diagnosable. We are done.

By Theorems 1 and 2, the following corollary holds.

Corollary 1. $t_{c}\left(L T Q_{n}\right)=4 n-7$ for $n \geq 5$.

\section{Conclusions}

Since the probability that any faulty set contains all the neighbors of some processor is very small, conditional diagnosability, requiring that each processor of a system is incident with at least one fault-free processor, can better measure the diagnosability of interconnection. In this paper, the main contribution is the determination of the conditional diagnosability of the locally twisted cubes. We obtain that the conditional diagnosability of a locally twisted cube under the PMC model is $t_{c}\left(L T Q_{n}\right)=4 n-$ 7 for $n \geq 5$.

\section{References}

[1] G. M. F. P. Preparata and R. T. Chien, "On the Connection Assignment Problem of Diagnosable Systems,” IEEE Transactions on Electronic Computers, Vol. EC-16, No. 6, December 1967, pp. 848-854. $\underline{\text { doi:10.1109/PGEC.1967.264748 }}$

[2] M. M. J. Maeng, "A Comparison Connection Assignment for Self-Diagnosis of Multiprocessors Systems,” Proceedings of the 11th International Symposium on FaultTolerant Computing, Portland, 1981, pp. 173-175.

[3] F. G. F. Barsi and P. Maestrini, "A Theory of Diagnosability of Digital Systems," IEEE Transactions on Computers, Vol. C-25, No. 6, June 1976, pp. 585-593. doi:10.1109/TC.1976.1674658

[4] R. Ahlswede and H. Aydinian, "On Diagnosability of Large Multiprocessor Networks," Discrete Applied Mathematics, Vol. 156, No. 18, 2008, pp. 3464-3474. doi:10.1016/j.dam.2008.02.001

[5] D. Wang, "Diagnosability of Enhanced Hypercubes," IEEE Transactions on Computers, Vol. 43, No. 9, 1994, pp. 10541061. doi:10.1109/12.312114

[6] J. Fan, "Diagnosability of the Mobius Cubes," IEEE Transactions on Parallel and Distributed Systems, Vol. 9, No. 9, 1998, pp. 923-928. doi:10.1109/71.722224
[7] P.-L. Lai, J. Tan, C.-P. Chang and L.-H. Hsu, “Conditional Diagnosability Measures for Large Multiprocessor Systems," IEEE Transactions on Computers, Vol. 54, No. 2, 2005, pp. 165-175. doi:10.1109/TC.2005.19

[8] S. Hsieh and C. Lee, "Diagnosability of Two-Matching Composition Networks under the MM* Model," IEEE Transactions on Dependable and Secure Computing, Vol. 8, No. 2, 2009, pp. 246-255.

[9] Q. Zhu, S.-Y. Liu and M. Xu, “On Conditional Diagnosability of the Folded Hypercubes,” Information Sciences, Vol. 178, No. 4, 2008, pp. 1069-1077. doi:10.1016/j.ins.2007.09.005

[10] M. Xu, K. Thulasiraman and X.-D. Hu, "Conditional Diagnosability of Matching Composition Networks under the Pmc Model," IEEE Transactions on Circuits and Systems II: Express Briefs, Vol. 56, No. 11, 2009, pp. 875- 879. doi:10.1109/TCSII.2009.2030361

[11] Q. Zhu, “On Conditional Diagnosability and Reliability of the bc Networks," The Journal of Supercomputing, Vol. 45, No. 2, 2008, pp. 173-184. doi:10.1007/s11227-007-0167-8

[12] S.-M. Zhou, “The Conditional Diagnosability of Locally Twisted Cubes," Proceedings of the 4th International Conference on Computer Science and Education, 2009, pp. 221-226.

[13] J. A. Bondy and U. S. R. Murty, "Graph Theory with Applications,” North Holland, New York, 1976.

[14] X.-F. Yang, D. J. Evans and G. M. Megson, "The Locally Twisted Cubes,” International Journal of Computer Mathematics, Vol. 82, No. 4, April 2005, pp. 401-413. doi:10.1080/0020716042000301752

[15] G. M. A. T. Dahbura, “An $\mathrm{O}\left(n^{2.5}\right)$ Fault Identification Algorithm for Diagnosable Systems,” IEEE Transactions on Computers, Vol. C-33, No. 6, 1984, pp. 486-492. doi:10.1109/TC.1984.1676472

[16] A. T. Dahbura and G. M. Masson, “An $O\left(n^{2.5}\right)$ Fault Identification Algorithm for Diagnosable Systems,” IEEE Trans- actions on Computers, Vol. 33, No. 6, 1984, pp. 486-492. doi:10.1109/TC.1984.1676472

[17] J.-X. Fan, S.-K. Zhang, et al., "The Restricted Connectivity of Locally Twisted Cubes,” 2009 10th International Symposium on Pervasive Systems, Algorithms, and Networks (ISPAN), Kaohsiung, 14-16 December 2009, pp. 574-578. doi:10.1109/I-SPAN.2009.48

[18] J. Fan and X. Lin, “The $t / k$-Diagnosability of the BC Graphs,” IEEE Transactions on Computers, Vol. 54, No. 2, 2005, pp. 176-184. doi:10.1109/TC.2005.33

[19] X.-F. Yang, J.-Q. Cao, G. M. Megson and J. Luo, "Minimum Neighborhood in a Generalized Cube,” Information Processing Letters, Vol. 97, 2006, pp. 88-93. 